\title{
What are the mechanisms for analogue and digital signalling in the brain?
}

\section{Dominique Debanne, Andrzej Bialowas and Sylvain Rama}

Abstract | Synaptic transmission in the brain generally depends on action potentials. However, recent studies indicate that subthreshold variation in the presynaptic membrane potential also determines spike-evoked transmission. The informational content of each presynaptic action potential is therefore greater than initially expected. The contribution of this synaptic property, which is a fast (from 0.01 to $10 \mathrm{~s}$ ) and state-dependent modulation of functional coupling, has been largely underestimated and could have important consequences for our understanding of information processing in neural networks. We discuss here how the membrane voltage of the presynaptic terminal might modulate neurotransmitter release by mechanisms that do not involve a change in presynaptic $\mathrm{Ca}^{2+}$ influx.

The principal function of the CNS is to adapt an organism's behaviour to changes in the environment - a process that is thought to be mediated by modulation of neuronal circuits through synaptic modifications. Understanding the cellular and molecular mechanisms underlying activity-dependent regulation of synaptic strength is therefore a major issue in neurobiology. Recent work indicates that in addition to changes in postsynaptic receptor expression and synaptic vesicle release probability, synaptic strength in mammalian neurons is regulated by subthreshold variations of voltage that are generated far from the synapse $\mathrm{e}^{1-4}$.

In most neurons, the proximal region of the axon (the axon initial segment (AIS)) contains a high density of $\mathrm{Na}^{+}$channels, and is therefore a hotspot for neuronal excitation. Small synaptic potentials that are generated in the dendrites summate temporally, and if the resulting potential reaches the threshold for action potential generation, a spike is initiated at the AIS. According to this view, the AIS is the final site for dendritic integration of synaptic responses ${ }^{5}$, which produces an action potential that is actively propagated along the axon. Neuronal information is therefore transmitted to the postsynaptic neuron as discrete amounts of neurotransmitter released by the presynaptic neuron in an all-or-none mode. This mode of neuronal signalling is thus 'digital': the neuron either fires or it does not, and neurotransmitter release follows this binary mode (FIG. 1a).

However, neuronal information is not only transmitted digitally, and subthreshold activity that originates in the dendrites and reaches the soma can be conveyed along the axon to the presynaptic element, in which it influences the arriving action potential and thus tunes the flow of neuronal information via 'analogue' coding. Clear examples of analogue transmission of neuronal information can be found in the inner ear or in the retina, in which photoreceptors, bipolar and horizontal cells signal photostimulation by producing graded potentials without action potentials ${ }^{6}$. These cells generally release transmitter continuously (tonic release), and their high rate of spontaneous release is directly modulated by membrane potential fluctuations (FIG. 1b). Similar examples of graded transmission in the absence of spiking activity have also been described in invertebrate neurons (see REF. 7 for a review).

There is now evidence that analogue signalling exists at spiking synapses, where neurotransmitter release is evoked by action potentials rather than being tonic. In this article, we discuss recent work suggesting the possibility of mixed analogue-digital signalling at local axonal connections in CNS circuits, with an emphasis on the hippocampus. This has important and exciting implications for our understanding of information processing in this part of the brain.

\section{Analogue and digital signalling}

Synapses translating analogue presynaptic membrane potential fluctuation into graded tonic release of transmitter display a significantly higher rate of information transfer than synapses using presynaptic spike train coding. The dynamic range at tonic-release, analogue synapses is indeed very large, and a single analogue synapse is able to continuously encode virtually infinite information levels. For instance, graded synapses in the fly retina transmit more than 1,500 bits of information per second: that is, one or two orders of magnitude larger than spiking neurons ${ }^{8,9}$. However, this comes at the price of high energy consumption. For example, photoreceptors in the retina continuously release their neurotransmitter at a high rate (20-80 vesicles per active zone per second), indicating that each active zone may release as many as a few million vesicles per day ${ }^{10}$. Another drawback of analogue signalling is that it is constrained by biophysical laws such as voltage dissipation along neuronal processes over long distances. Therefore, pure analogue signalling in neurons is better suited for local rather than distal transmission of information.

By contrast, digital synapses are able to signal activity far from the site of spike initiation because neuronal information encoded in the form of action potentials is carried over very long distances along axons without voltage dissipation, as active currents regenerate action potentials along the axon ${ }^{11,12}$. Another major advantage of 


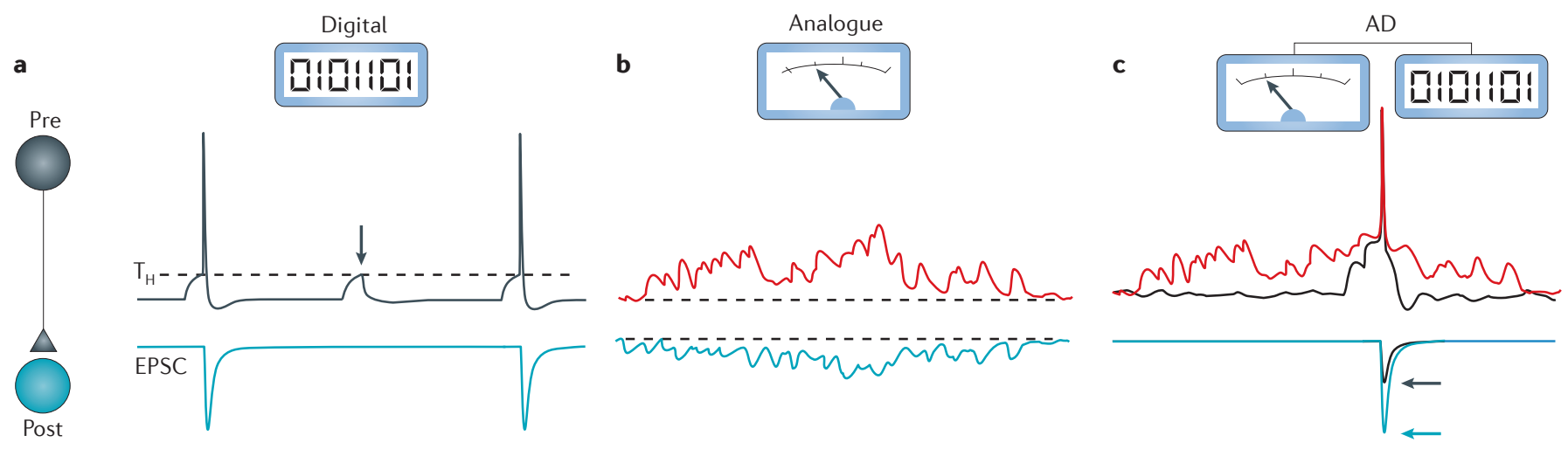

d

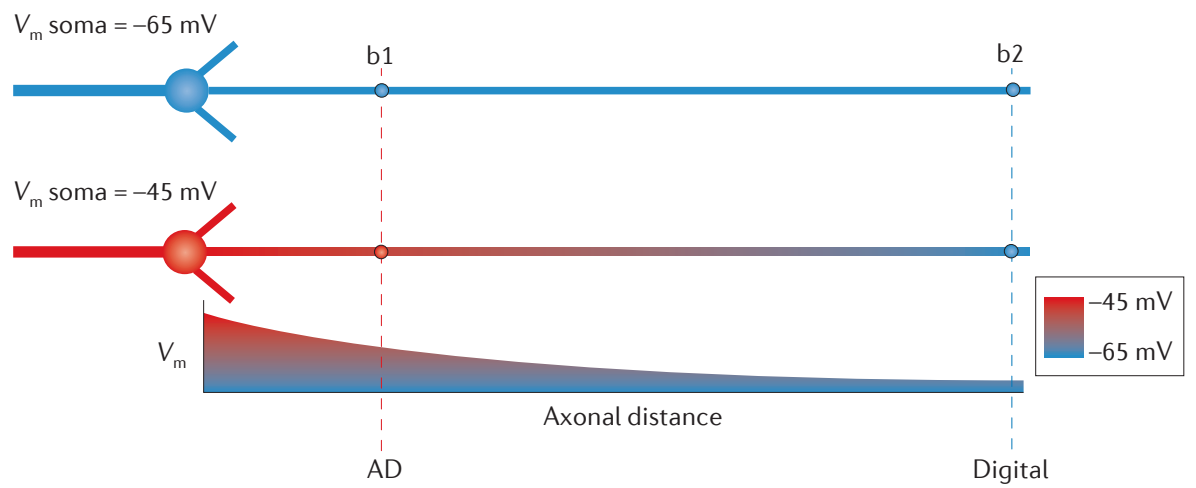

Figure 1 | Digital, analogue and hybrid (analogue-digital) modes of synaptic transmission. a| Digital mode of synaptic transmission in the CNS. A scheme of two synaptically connected neurons is shown on the left. Transmission (right) is stereotyped and occurs in an all-or-none (digital) manner (that is, only if a presynaptic action potential is elicited). Note that subthreshold depolarization (indicated by the arrowhead) produces neither a presynaptic spike nor a postsynaptic response. $\mathbf{b} \mid$ Analogue transmission is a graded mode of transmission of presynaptic voltage fluctuations. The two horizontal dashed lines indicate the baselines. The blue trace represents postsynaptic activity.

c| Hybrid analogue-digital (AD) transmission. Both subthreshold fluctuations (red trace) and spiking activity (upper black trace) are transmitted. Note that when the presynaptic spike is produced after a prolonged period of depolarization (red trace), the spike-evoked synaptic response (blue trace) is enhanced compared with when there is no prolonged depolarization (upper and lower black traces). d | Spatial gradient of hybrid and digital transmission. Because of cable properties, AD transmission is restricted to proximal presynaptic boutons (b1), whereas pure digital transmission occurs at distal boutons (b2). EPSC, excitatory postsynaptic current; $T_{H}$, spike threshold; $V_{m}$, membrane voltage.

digital signalling is its relatively low energy cost. The kinetics of voltage-gated currents underlying the action potential are tuned to minimize energetic consumption ${ }^{13,14}$. Therefore, in many ways, digital signalling may appear to be an improvement on analogue signalling. However, it has also several limitations. Because of its discrete nature, the coding of information by a single digital synapse is generally poor in comparison with analogue synapses. Thus, combining analogue and digital signalling should in principle offer a balance between the dual advantages low energy cost and highly dynamic transfer of neuronal information.

Hybrid analogue-digital enhancement of synaptic transmission at spiking synapses that was initially described in invertebrates ${ }^{15-18}$ has more recently been reported in many mammalian CNS synapses, including cortical ${ }^{2,3,19}$, cerebel$\operatorname{lar}^{20,21}$ and hippocampal synapses ${ }^{1,22}$. In these examples, synaptic transmission evoked by single action potentials is enhanced as the result of analogue-mediated depolarization $(10-30 \mathrm{mV})$ of the presynaptic element for a few tens to hundreds of milliseconds (FIG. 1c).

\section{Voltage propagation in axons}

A prerequisite for analogue-digital facilitation $(\mathrm{ADF})$ is that analogue voltage changes produced in the somatodendritic regions are capable of spreading along the axon over long enough distances to reach synapses. This process of analogue voltage propagation is based on the electrical properties of the axon membrane. It was first theorized by Rall ${ }^{23}$ who modelled the axon as a 'membrane cylinder' and showed that a voltage response decays exponentially along passive cables. The space constant is the axonal distance for which voltage drops to $37 \%$ of its initial value. It depends on both geometrical and electrical factors of the axon: the axon membrane resistance, which is determined by the relative contribution of conducting (pore-forming proteins) and non-conducting (lipids) molecules, and axial resistance, which is controlled by the intra-axonal medium and the axon diameter. Thus, a large space constant is generally obtained for low intra-axonal resistance or high axonal membrane resistance. Excitatory postsynaptic potentials (EPSPs) that are generated in the dendritic tree of hippocampal dentate granule cells travel passively towards the soma and along the axon, and the resulting excitatory presynaptic potential (EPreSP) in mossy fibre terminals can be 
measured with a patch-pipette ${ }^{1}$. The axonal space constant for this transient depolarizing event ( $50-100 \mathrm{~ms})$ has been estimated as $\sim 450 \mu \mathrm{m}^{1}$. The space constant varies as a function of the frequency, diminishing rapidly at high frequency (see supplementary information in REF. 2). In neocortical pyramidal neurons, the axonal space constant of steady-state voltage modulation in the soma yields values of $420-550 \mu \mathrm{m}^{2,3}$. In principle, active conductance such as a persistent $\mathrm{Na}^{+}$current could enhance the axon space constant by carrying subthreshold depolarization that is initiated in the soma over longer axonal distances. However, the large space constant of EPreSPs in hippocampal granule cell axons does not result from the activation of $\mathrm{Na}^{+}$channels because it is unaffected by tetrodotoxin ${ }^{1}$. Therefore, the large space constant of granule cell axons may result from specific electrical properties (that is, high membrane resistance and/or low axial resistance) that need to be identified.

The notion of an axonal space constant is crucial for analogue-digital signalling because it describes how voltage spreads along the axon and modulates the biophysical properties of ion channels located in presynaptic terminals. This modulation depends on the gating characteristics of the current. For instance, a modest subthreshold depolarization will be more effective on voltage-gated currents with low activationinactivation thresholds (that is, near the resting membrane potential) than on those with high activation-inactivation thresholds (that is, far from the resting membrane potential). Consequently, ADF will spread over long distances if the underlying mechanism involves a low threshold conductance.

As many CNS neurons establish local recurrent connections, analogue-digital enhancement is therefore likely to have a major role in a potentially large number of cortical, hippocampal and cerebellar excitatory and inhibitory circuits ${ }^{2,3,19,20}$. An important consequence of the size of the axon space constant is that pure digital and hybrid signalling may coexist within the same axon. In cortical pyramidal cell axons, local connections established by boutons located in the proximal bouquet of axon collaterals are likely to function in a hybrid analogue-digital way, whereas long projecting axon collaterals may essentially work under a digital mode (FIG. 1d). For example, at excitatory connections between layer 5 (L5) pyramidal neurons, the larger ADF is observed at local connections ${ }^{3}$. In addition, a similar spatial dichotomy has recently been reported in CA3 pyramidal neurons in which ADF is observed at proximal synapses established with other CA3 neurons but not at synapses with distal CA1 neurons $^{24}$. In this way, analogue-digital signalling increases the computational repertoire of neuronal communication.

\section{Mechanisms of ADF}

Principles of ADF. ADF occurs in a highly heterogeneous range of synapses in terms of morphology (en passant boutons and giant terminals), neurotransmitter (GABA or glutamate) and brain regions (neocortex, hippocampus and cerebellum). ADF involves two major steps: the depolarization of the presynaptic element, which then causes the enhancement of neurotransmitter release.

In cerebellar synapses established between GABAergic interneurons of the molecular layer, subthreshold depolarization facilitates spike-evoked release of $\mathrm{GABA}^{20}$. In hippocampal dentate granule cell axons (mossy fibres), the combination of analogue depolarization spreading from the soma in the form of an EPreSP and digital signalling in the form of an action potential, enhances glutamatergic transmission at the mossy fibre-CA3 cell synapse $^{1}$. For local connections such as these that occur over relatively short distances between L5 pyramidal neurons in the neocortex or GABAergic interneurons in the cerebellum, the analogue-mediated component of the facilitation of synaptic transmission is on average $1-2 \%$ per $\mathrm{mV}$ of somatic depolarization ${ }^{2,3,20}$.

$\mathrm{ADF}$ is mediated by an increase in glutamate ${ }^{3,25}$ or $\mathrm{GABA}^{20,21}$ release, as indicated by the reduced paired-pulse ratio (PPR): that is, the ratio of synaptic responses for a pair of presynaptic stimuli. Intriguingly, however, in the case of the hippocampal mossy fibre-CA3 cell synapse, short-term facilitation tested with repeated presynaptic stimuli is unchanged ${ }^{1}$, suggesting that (as discussed later) glutamate release is not changed in a conventional way.

Although there are general principles on which ADF mechanisms operate at synapses, important differences also exist. For instance, the temporal requirement for $\mathrm{ADF}$ is highly heterogeneous. In L5 pyramidal neurons, ADF is observed after several seconds of presynaptic depolarization $^{2,3}$, whereas a few tens of milliseconds of analogue subthreshold depolarization is sufficient to induce ADF at mossy fibre-CA3 cell synapses ${ }^{1}$ or at cerebellar GABAergic synapses ${ }^{21}$.
Inactivation of presynaptic $K^{+}$channels. So far, two independent mechanisms have been reported to account for the analoguedigital enhancement of transmission in central synapses. First, depolarization of the somatic region of the presynaptic neuron may enhance synaptic transmission as the consequence of voltage-inactivation of a specific type of $\mathrm{K}^{+}$channel (FIG. 2a). Axons contain a high density of voltagegated $\mathrm{K}^{+}\left(\mathrm{K}_{\mathrm{v}}\right)$ channels. They generate a current that is strongly inactivated by a small depolarization, suggesting that its contribution to ADF will be important even in distal regions of the axon, in which analogue subthreshold depolarization is minimal. In neocortical and hippocampal pyramidal neurons, for example, the $\mathrm{K}_{\mathrm{V}} 1$ channels generate a fast-activating but slowly-inactivating $\mathrm{D}$-type current $\left(I_{\mathrm{D}}\right)$ that reduces spike duration and thus controls neurotransmitter release $\mathrm{e}^{3,26-28}$. Pharmacological inactivation of $I_{\mathrm{D}}$ with 4-aminopyridine or dendrotoxin enhances synaptic strength at hippocampal and neocortical synapses possibly by broadening the presynaptic spike in the terminal, resulting in higher release of neurotransmitter $^{3,28,29}$. In a similar manner, blocking $I_{\mathrm{D}}$ in cortical pyramidal cells also broadens the spike in the axon and at presynaptic terminals ${ }^{3,28,30}$ and occludes ADF of synaptic strength ${ }^{3}$. Furthermore, the kinetics of the analogue-digital enhancement fit well with the inactivation kinetics of $I_{\mathrm{D}}{ }^{2,3}$. Last, voltage inactivation of $\mathrm{K}_{\mathrm{V}} 1$ channels causes a significant enhancement of the $\mathrm{Ca}^{2+}$ transient in the presynaptic terminal that is evoked by the propagating spike in the presynaptic terminal ${ }^{31}$. In fact, $\mathrm{Ca}^{2+}$ chelation with EGTA (ethylene glycol tetra-acetic acid) abolishes ADF in L5 pyramidal neurons $^{2}$. Enhanced neurotransmitter release by the inactivation of $\mathrm{K}_{\mathrm{v}} 1$ channels is not solely mediated by intrinsic mechanisms (that is, depolarization of the presynaptic neuron). In fact, extrinsic signals, such as glutamate released from astrocytes, that are present abundantly adjacent to hippocampal axons and their terminals can also modulate the width of action potentials in axons and enhance synaptic transmission in an analogue manner ${ }^{32,33}$.

Increase of basal $\mathrm{Ca}^{2+}$ concentration.

Propagated depolarization can also facilitate synaptic transmission by the opening of voltage-gated $\mathrm{Ca}^{2+}\left(\mathrm{Ca}_{\mathrm{v}}\right)$ channels, which results in an increase in basal $\mathrm{Ca}^{2+}$ concentration in presynaptic terminals (FIG. 2b). At rest (that is, $-65 \mathrm{mV}$ ), intraterminal $\mathrm{Ca}^{2+}$ 

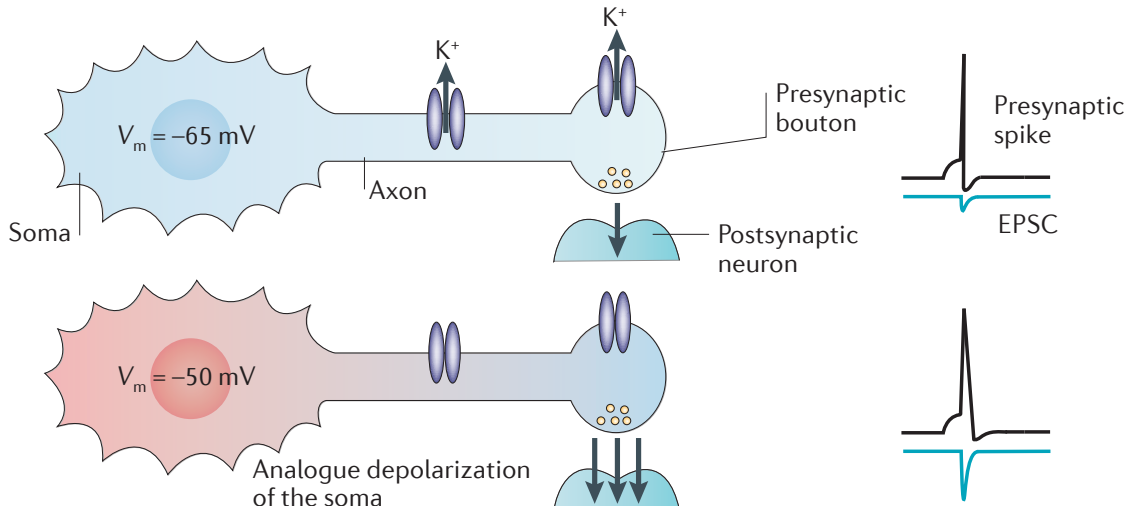

b
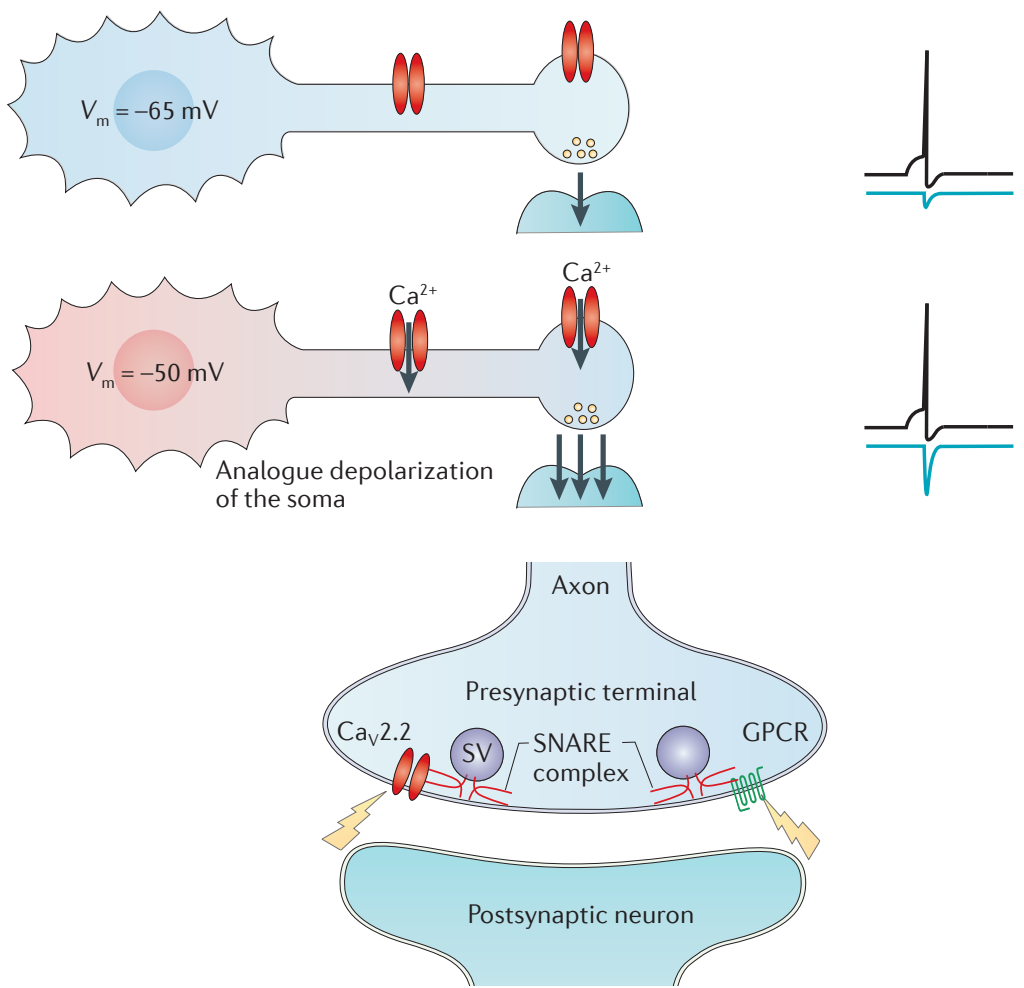

Figure 2 | Generic mechanisms for analogue-digital facilitation. a | Facilitation resulting from voltage-inactivation of voltage-gated $K_{v} 1$ channels. With digital-only signalling (upper neuron), the neuronal membrane voltage $\left(V_{m}\right)$ is held at $-65 \mathrm{mV}$ and $K_{v} 1$ channels remain open, resulting in a rapid termination of the spike response. Analogue subthreshold depolarization (lower neuron; $-50 \mathrm{mV}$ at the soma) propagates from the somatic compartment and along the axon to the terminal (the gradual decay in depolarization is indicated by red-to-blue shading), resulting in closure of $\mathrm{K}_{\mathrm{v}} 1$ channels and attenuated $\mathrm{K}^{+}$efflux. This produces a broader action potential and results in enhanced spike-evoked $\mathrm{Ca}^{2+}$ entry and incremented neurotransmitter release (single black arrow). $\mathbf{b}$ | Facilitation of transmission resulting from an increase of basal $\mathrm{Ca}^{2+}$ concentration. Under digital signalling conditions (upper neuron; $\left.V_{m}=-65 \mathrm{mV}\right)$, the arrival of an action potential is insufficient to open voltage-gated $\mathrm{Ca}^{2+}\left(\mathrm{Ca}_{\mathrm{v}}\right)$ channels (shown in red) but is sufficient to evoke release of neurotransmitter (black arrow). During analogue-digital signalling (lower neuron), partial depolarization $\left(V_{m}=-50 m V\right)$ spreading from the soma opens $\mathrm{Ca}_{V}$ channels, increases $\mathrm{Ca}^{2+}$ influx and basal $\mathrm{Ca}^{2+}$ concentration in the presynaptic bouton and enhances neurotransmitter release (indicated by three black arrows) when a presynaptic action potential also arrives at the bouton. $\mathbf{c}$ |Two putative mechanisms of enhanced exocytosis by neuronal $V_{m}$. On the left, a subthreshold change in $V_{m}$ (lightning bolt) is detected by the voltage sensor of $\mathrm{Ca}_{\mathrm{v}} 2.2$ channel (shown in red) that interacts with SNARE (soluble NSF ( $N$-ethylmaleimide-sensitive factor) attachment protein receptor) proteins (which are part of the exocytosis machinery for synaptic vesicles (SVs)). This results in facilitation of SV fusion and enhanced transmitter release. On the right, a subthreshold change in $V_{m}$ is detected by $G$ protein-coupled receptors (GPCRs), such as type 3 metabotropic glutamate receptor, which interact with the release machinery to influence neurotransmitter release. EPSC, excitatory postsynaptic current. concentration is $\sim 100 \mathrm{nM}$ and can increase up to $1 \mu \mathrm{M}$ upon subthreshold depolarization $\left(\sim-50 \mathrm{mV}^{25}\right)$. This increase can easily be measured in thin cerebellar axons using fluorescent $\mathrm{Ca}^{2+}$ probes ${ }^{20,21}$. Alternatively, $\mathrm{Ca}^{2+}$ currents can be directly monitored with patch-clamp recording from large terminals such as the calyx of Held ${ }^{25}$. There is a clear consensus among studies in various brain areas that the $\mathrm{Ca}_{v}$ channels responsible for this facilitation, whether in the axon terminal ${ }^{20,21,25}$ or in the axon itself ${ }^{31}$, are $\mathrm{Ca}_{\mathrm{v}} 2.1$ channels (also known as P/Qtype $\mathrm{Ca}^{2+}$ channels). $\mathrm{Ca}_{\mathrm{v}} 2.2$ channels (also known as $\mathrm{N}$-type $\mathrm{Ca}^{2+}$ channels) that are located in the axon terminal and the AIS also participate in the increase in baseline $\mathrm{Ca}^{2+}$ signal upon depolarization ${ }^{31} \cdot \mathrm{Ca}_{\mathrm{v}} 2.1$ and $\mathrm{Ca}_{\mathrm{v}} 2.2$ channels are activated by high levels of depolarization. Thus, because of voltage attenuation along the axon, their contribution to ADF might be limited to very proximal inputs. The hippocampal mossy fibre terminal, however, appears to be an exception, as subthreshold depolarization induces a $\mathrm{Ca}^{2+}$ influx that is not only mediated by the classical $\mathrm{Ca}_{\mathrm{v}} 2.1$ and $\mathrm{Ca}_{\mathrm{v}} 2.2$ channels but also by $\mathrm{Ca}_{\mathrm{v}} 2.3$ channels (also known as R-type $\mathrm{Ca}^{2+}$ channels) $)^{34}$.

The increase in basal $\mathrm{Ca}^{2+}$ concentration triggered by subthreshold depolarization could have two major consequences. First, it might directly promote neurotransmitter release. In axons of cerebellar interneurons, for example, subthreshold depolarization is sufficient to increase spontaneous, spike-independent release of $\mathrm{GABA}^{20}$. A second possible action of an increase in basal $\mathrm{Ca}^{2+}$ concentration is an acceleration of the recruitment of vesicles to the active zone ${ }^{35}$. The principal consequence of this $\mathrm{Ca}^{2+}$-induced priming of vesicles would be an increase in synchronous release when the presynaptic spike invades the axon terminal. Both of these effects would result in an increase in neurotransmitter release and enhanced synaptic strength.

\section{Unsolved question: does ADF occur at the hippocampal mossy fibre synapse? The} cellular mechanisms underlying analoguedigital enhancement of synaptic strength at mossy fibre synapses with CA3 cells challenge the classical view of synaptic strength modulation. First, unlike most examples of analogue-digital enhancement, the PPR is unaffected despite excitatory postsynaptic current (EPSC) facilitation of $\sim 40 \%$ in the CA3 cell ${ }^{1}$. One explanation for this is that the low release probability of glutamate 


\section{Box 1 | Relationship between $\mathrm{Ca}^{2+}$ and exocytosis}

$\mathrm{Ca}^{2+}$ ions are required for exocytosis. However the relationship between the presynaptic $\mathrm{Ca}^{2+}$ charge (that is, the total amount of $\mathrm{Ca}^{2+}$ ions) and the exocytosis is not linear. At many CNS synapses, the postsynaptic current (PSC) can be described by a power law (PSC $=a x^{m}$, where $x$ is the presynaptic $\mathrm{Ca}^{2+}$ concentration, $a$ is a constant and $m$ is the power coefficient). The power coefficient is an indicator of the cooperativity of $\mathrm{Ca}^{2+}$ binding to presynaptic $\mathrm{Ca}^{2+}$ sensor proteins of the vesicle release machinery and varies considerably from synapse to synapse. For instance, in hippocampal basket cells, this coefficient is 1.6, indicating relatively low cooperativity at the output synapses of hippocampal basket cells ${ }^{65}$, but it may reach 5 at the synapse formed by the calyx of Held ${ }^{66}$. In this latter case, very small variations in presynaptic $\mathrm{Ca}^{2+}$ concentration resulting from small subthreshold analogue modulation of the spike waveform may lead to an extremely large increase in neurotransmitter release. Thus, analogue-digital modulation of synaptic transmission might be extremely heterogeneous among synapses.

$(\sim 5 \%)$ at this synapse would produce little vesicle depletion, and thus although more glutamate is being released (accounting for the enhanced EPSC), there would be no detectable change in the PPR. This hypothesis is plausible but, conversely, relatively modest synaptic enhancements, such as those produced by LTP $(\sim 50 \%)$, result in clear changes in the PPR at the mossy fibre synapse $^{36-38}$. An alternative explanation, which is supported by a number of findings, is that analogue-digital enhancement at mossy fibre synapses might not entirely result from a classical rise in presynaptic neurotransmitter release. For example, maximal subthreshold depolarization of the mossy fibre terminal in the hippocampus produces no detectable change in presynaptic intracellular $\mathrm{Ca}^{2+}$ signals ${ }^{39}$. Moreover, subthreshold depolarization of the granule cell soma affects $\mathrm{Ca}^{2+}$ signalling only in proximal segments of granule cell axons, suggesting that analogue-digital enhancement at more distal mossy fibre boutons might be largely independent of $\mathrm{Ca}^{2+}$. One may argue that the sensitivity of the fluorescent $\mathrm{Ca}^{2+}$ probe might not be sufficient to detect very small $\mathrm{Ca}^{2+}$ increases in cellular nanodomains ${ }^{39,40}$. Supporting this view, analogue-digital enhancement at the mossy fibre synapse is partly reduced by buffering $\mathrm{Ca}^{2+}$ with EGTA ${ }^{1}$.

How could enhanced release be produced without any change in the spikeevoked $\mathrm{Ca}^{2+}$ transient? The classical view that presynaptic facilitation is necessarily associated with the modulation of the presynaptic $\mathrm{Ca}^{2+}$ signals (BOX 1) has been convincingly challenged by Felmy and co-workers ${ }^{41}$. They showed that a threefold increase in synaptic strength can be induced at the calyx of Held synapse by a prolonged subthreshold depolarization without any change in $\mathrm{Ca}^{2+}$ during the presynaptic spike. In this case, $\mathrm{Ca}^{2+}$ concentration is raised in the calyx of Held during the subthreshold depolarization, indicating that, at this synapse, facilitation results from the supra-linear summation of intracellular $\mathrm{Ca}^{2+}$ signals arising from different sources ${ }^{41}$.

In summary, one cannot totally exclude that analogue-digital enhancement at the mossy fibre synapse results from an undetectable $\mathrm{Ca}^{2+}$ rise produced by the EPreSP that causes supra-linear summation between conditional (analogue depolarization) and spike-evoked $\mathrm{Ca}^{2+}$ signals. However, this solution is still not entirely satisfying because, in contrast with experimental results ${ }^{1}$, short-term facilitation should be reduced in this case, so other mechanisms must be considered.

\section{Modulation of release by voltage} Do $\mathrm{Ca}^{2+}$ channels act as voltage sensors in presynaptic terminals? Voltage can also induce transmitter release by a direct, $\mathrm{Ca}^{2+}$ independent effect on release machinery. Several parallel studies have reported facilitation of synaptic transmission that is produced by subthreshold depolarization of the presynaptic terminal ${ }^{42-44}$. Furthermore, in a few rare cases, such as in dorsal root ganglion neurons, transmitter release is $\mathrm{Ca}^{2+}$ independent but voltage-dependent ${ }^{45}$. The underlying mechanisms in this particular case remain unknown, but at superior cervical ganglion synapses, the synaptic protein interaction site of $\mathrm{Ca}_{\mathrm{v}} 2.2$ channels that are present on the presynaptic membrane and adjacent to synaptic vesicle release sites mediates a voltage-dependent facilitation of transmission ${ }^{46}$. In this case, presynaptic depolarization is likely to be mediated by the voltage sensor of the $\mathrm{Ca}_{\mathrm{v}} 2.2$ channel and directly transmitted to the SNARE (soluble NSF ( $N$-ethylmaleimide-sensitive factor) attachment protein receptor) proteins (FIG. 2c). This mechanism could mediate analogue-digital enhancement at mossy fibre boutons because $\mathrm{Ca}_{\mathrm{v}} 2.2$ channels are broadly expressed at CNS terminals and particularly at mossy fibre boutons ${ }^{34}$.

The direct contribution of presynaptic voltage has been tested at the calyx of Held synapse where the release rate increased only slightly (by 20\%) when the presynaptic terminal was depolarized by $+80 \mathrm{mV}$ during $\mathrm{Ca}^{2+}$ uncaging ${ }^{47}$. Smaller levels of depolarization (from +10 to $+50 \mathrm{mV}$ ) were not tested in this study, so additional experiments will be necessary to clarify this point.

Other voltage-gated channels such as $\mathrm{K}_{\mathrm{V}} 1.1$ and $\mathrm{K}_{\mathrm{v}} 2.1$ interact with presynaptic SNARE proteins and facilitate neurotransmission $^{48-50}$, but it is not yet clear whether detection of voltage by their voltage sensors has a critical role in this facilitation.

Do G protein-coupled receptors act as voltage sensors at presynaptic terminals? Another mechanism of voltage-dependent facilitation of exocytosis has been proposed at cholinergic synapses ${ }^{51}$. Because of charge movement, binding of acetylcholine to the M2 muscarinic G protein-coupled receptor (GPCR) is strongly modulated by membrane potential $^{52}$. The GPCR is bound to the release machinery at rest, producing a tonic inhibition of transmitter release, but is unbound when there is a depolarized potential ${ }^{53,54}$ (FIC. 2c). The functional consequence of the depolarization produced by the presynaptic spike would be a facilitation of transmitter release. A similar mechanism might operate at glutamatergic synapses as the kinetics of glutamate release at the neuromuscular junction of the crayfish are also controlled by $\mathrm{GPCRs}^{55}$, and presynaptic metabotropic glutamate receptors are voltage-sensitive ${ }^{56}$. The mossy fibre terminal contains a wide range of GPCRs, including type B GABA receptors ${ }^{57}$, type 2 and 3 metabotropic glutamate receptors $^{58}$ and adenosine $\mathrm{A} 1$ receptors ${ }^{59}$. However, the contribution of GPCRs to analoguedigital enhancement of transmission at the mossy fibre synapse remains to be precisely determined.

It is important to note that even if release modulation could result from a direct action of voltage on $\mathrm{Ca}^{2+}$ channels or GPCRs, the PPR would still be modulated because vesicle depletion would still occur. Therefore, the lack of PPR modulation observed at mossy fibre synapse cannot be satisfactorily explained by this mechanism.

\footnotetext{
Does voltage modulate the ratio of kissand-run versus full-fusion exocytosis? Whether other molecular mechanisms of $\mathrm{Ca}^{2+}$-independent and voltage-dependent exocytosis contribute to voltage-dependent
} 
modulation of the release machinery remains unknown. However, one may speculate that transmitter release might theoretically be enhanced if the relative ratio of kiss-and-run versus full-fusion mode of exocytosis decreases after analogue depolarization $^{60}$. The kiss-and-run mode of exocytosis corresponds to an incomplete fusion of the presynaptic vesicle by which only a small fraction of its content is released ${ }^{60}$. Thus, compared with the full-fusion mode of release, synaptic transmission is not maximal because not all postsynaptic receptors are stimulated. Kiss-and-run exocytosis has been reported in cultured hippocampal neurons ${ }^{61,62}$, but it has not yet been demonstrated at the mossy fibre synapse. It is noteworthy that if neurotransmitter release was enhanced in $\mathrm{ADF}$ through a decrease in the kiss-and-run mode of fusion, release probability and the PPR would not change, as seen at the mossy fibre synapse.

The $\beta \gamma$ subunits of $\mathrm{G}$ proteins are present in a wide range of presynaptic terminals, including mossy fibre synapses, are thought to influence the opening of the fusion pore downstream of $\mathrm{Ca}^{2+}$ entry ${ }^{63,64}$; however, the precise role of voltage in this process remains unknown. One may speculate that modifications of the open time of the fusion pore may not affect the PPR in a classical way. Additional experimental studies will be needed to determine whether and how subthreshold depolarization may modulate kiss-and-run exocytosis at central synapses.

\section{Conclusion}

In summary, synaptic transmission is highly modulated by changes in analogue voltage resulting from synaptic activity that is generated in the dendrites of CNS neurons. This form of activity-dependent regulation of synaptic strength is computationally appealing because many cortical neurons establish local connections through proximal synaptic inputs. Two principal presynaptic mechanisms have been identified in the recent years: a spikewidth-dependent facilitation and a basal $\mathrm{Ca}^{2+}$-dependent enhancement. However, analogue-digital enhancement of transmission at mossy fibre synapses may also involve other processes. Alternative mechanisms to the classical $\mathrm{Ca}^{2+}$-induced modulation of exocytosis have been suggested, such as $\mathrm{Ca}^{2+}$-independent but voltagedependent exocytosis. However, the role of this mechanism remains controversial, and its precise contribution to analogue-digital enhancement has yet to be identified.
Dominique Debanne, Andrzej Bialowas and Sylvain Rama are at INSERM, UMR_S 1072, and Aix-Marseille Université, UNIS, 13015, Marseille, France. Correspondence to D.D. e-mail:dominique.debanne@univ-amu.fr

1. Alle, H. \& Geiger, J. R. Combined analog and action potential coding in hippocampal mossy fibers. Science 311, 1290-1293 (2006).

2. Shu, Y., Hasenstaub, A., Duque, A., Yu, Y. \& McCormick, D. A. Modulation of intracortical synaptic potentials by presynaptic somatic membrane potential. Nature 441, 761-765 (2006).

3. Kole, M. H., Letzkus, J. J. \& Stuart, G. J. Axon initial segment Kv1 channels control axonal action potential waveform and synaptic efficacy. Neuron 55, 633-647 (2007).

4. Alle, H. \& Geiger, J. R. Analog signalling in mammalian cortical axons. Curr. Opin. Neurobiol. 18, 314-320 (2008).

5. Kole, M. H. \& Stuart, G. J. Signal processing in the axon initial segment. Neuron 73, 235-247 (2012).

6. Werblin, F. S. \& Dowling, J. E. Organization of the retina of the mudpuppy, Necturus maculosus. II. Intracellular recording. J. Neurophysiol. 32, 339-355 (1969).

7. Marder, E. Neurobiology: extending influence. Nature 441, 702-703 (2006)

8. de Ruyter van Steveninck, R. R. \& Laughlin, S. B. The rate of information transfer at graded-potential synapses. Nature 379, 642-645 (1996).

9. Borst, A. \& Theunissen, F. E. Information theory and neural coding. Nature Neurosci. 2, 947-957 (1999).

10. Heidelberger, R. Mechanisms of tonic, graded release: lessons from the vertebrate photoreceptor. J. Physiol. 585, 663-667 (2007).

11. Debanne, D., Campanac, E., Bialowas, A., Carlier, E. \& Alcaraz, G. Axon physiology. Physiol. Rev. 91 , 555-602 (2011).

12. Bucher, D. \& Goaillard, J. M. Beyond faithful conduction: short-term dynamics, neuromodulation, and long-term regulation of spike propagation in the axon. Prog. Neurobiol. 94, 307-346 (2011).

13. Alle, H., Roth, A. \& Geiger, J. R. Energy-efficient action potentials in hippocampal mossy fibers. Science $\mathbf{3 2 5}$, 1405-1408 (2009).

14. Sengupta, B., Stemmler, M., Laughlin, S. B. \& Niven, J. E. Action potential energy efficiency varies among neuron types in vertebrates and invertebrates. PLoS Comput. Biol. 6, e 1000840 (2010).

15. Shimahara, T. \& Tauc, L. Multiple interneuronal afferents to the giant cells in Aplysia. J. Physiol. 247, 299-319 (1975).

16. Shimahara, T. \& Peretz, B. Soma potential of an interneurone controls transmitter release in a monosynaptic pathway in Aplysia. Nature 273. 158-160 (1978)

17. Nicholls, J. \& Wallace, B. G. Modulation of transmission at an inhibitory synapse in the central nervous system of the leech. J. Physiol. 281, 157-170 (1978)

18. Shapiro, E., Castellucci, V. F. \& Kandel, E. R. Presynaptic membrane potential affects transmitter release in an identified neuron in Aplysia by modulating the $\mathrm{Ca}^{2+}$ and $\mathrm{K}^{+}$currents. Proc. Natl Acad. Sci. USA 77, 629-633 (1980).

19. Zhu, J., Jiang, M., Yang, M., Hou, H. \& Shu, Y. Membrane potential-dependent modulation of recurrent inhibition in rat neocortex. PLOS Biol. 9 e1001032 (2011).

20. Christie, J. M., Chiu, D. N. \& Jahr, C. E. Ca ${ }^{2+}$-dependent enhancement of release by subthreshold somatic depolarization. Nature Neurosci. 14, 62-68 (2011).

21. Bouhours, B., Trigo, F. F. \& Marty, A. Somatic depolarization enhances GABA release in cerebellar interneurons via a calcium/protein kinase $C$ pathway. J. Neurosci. 31, 5804-5815 (2011).

22. Debanne, D., Guerineau, N. C., Gahwiler, B. H. \& Thompson, S. M. Action-potential propagation gated by an axonal I-like $\mathrm{K}^{+}$conductance in hippocampus. Nature 389, 286-289 (1997).

23. Rall, W. Distributions of potential in cylindrical coordinates and time constants for a membrane cylinder. Biophys. J. 9, 1509-1541 (1969).

24. Sasaki, T., Matsuki, N. \& Ikegaya, Y. Effects of axonal topology on the somatic modulation of synaptic outputs. J. Neurosci. 32, 2868-2876 (2012).
25. Awatramani, G. B., Price, G. D. \& Trussell, L. O. Modulation of transmitter release by presynaptic resting potential and background calcium levels. Neuron 48, 109-121 (2005).

26. Shu, Y., Yu, Y., Yang, J. \& McCormick, D. A. Selective control of cortical axonal spikes by a slowly inactivating $\mathrm{K}^{+}$current. Proc. Natl Acad. Sci. USA 104, 11453-11458 (2007)

27. Shu, Y., Duque, A., Yu, Y., Haider, B. \& McCormick, D. A Properties of action-potential initiation in neocortical pyramidal cells: evidence from whole cell axon recordings. J. Neurophysiol. 97, 746-760 (2007).

28. Boudkkazi, S., Fronzaroli-Molinieres, L. $\&$ Debanne, D. Presynaptic action potential waveform determines cortical synaptic latency. J. Physiol. 589, 1117-1131 (2011).

29. Saviane, C., Mohajerani, M. H. ¿ Cherubini, E. An $\mathrm{I}_{\mathrm{D}}$-like current that is downregulated by $\mathrm{Ca}^{2+}$ modulates information coding at CA3-CA3 synapses in the rat hippocampus. J. Physiol. 552, 513-524 (2003).

30. Foust, A. J., Yu, Y., Popovic, M., Zecevic, D. \& McCormick, D. A. Somatic membrane potential and Kv 1 channels control spike repolarization in cortical axon collaterals and presynaptic boutons. J. Neurosci. 31, 15490-15498 (2011).

31. Yu, Y., Maureira, C., Liu, X. \& McCormick, D. P/Q and $\mathrm{N}$ channels control baseline and spike-triggered calcium levels in neocortical axons and synaptic boutons. J. Neurosci. 30, 11858-11869 (2010).

32. Sasaki, T., Matsuki, N. \& Ikegaya, Y. Action-potential modulation during axonal conduction. Science 331 599-601 (2011).

33. Debanne, D. \& Rama, S. Astrocytes shape axonal signaling. Sci. Signal. 4, pe11 (2011).

34. Li, L., Bischofberger, J. ¿ Jonas, P. Differential gating and recruitment of P/Q-, $\mathrm{N}-$, and $\mathrm{R}$-type $\mathrm{Ca}^{2+}$ channels in hippocampal mossy fiber boutons. J. Neurosci. 27, 13420-13429 (2007).

35. Neher, E. \& Sakaba, T. Multiple roles of calcium ions in the regulation of neurotransmitter release. Neuron 59, 861-872 (2008)

36. Zalutsky, R. A. \& Nicoll, R. A. Comparison of two forms of long-term potentiation in single hippocampal neurons. Science 248, 1619-1624 (1990).

37. Salin, P. A., Malenka, R. C. \& Nicoll, R. A. Cyclic AMP mediates a presynaptic form of LTP at cerebellar parallel fiber synapses. Neuron 16, 797-803 (1996)

38. Alle, H., Jonas, P. \& Geiger, J. R. PTP \& LTP at a hippocampal mossy fiber-interneuron synapse. Proc. Natl Acad. Sci. USA 98, 14708-14713 (2001).

39. Scott, R., Ruiz, A., Henneberger, C., Kullmann, D. M. $\&$ Rusakov, D. A. Analog modulation of mossy fiber transmission is uncoupled from changes in presynaptic $\mathrm{Ca}^{2+}$. J. Neurosci. 28, 7765-7773 (2008).

40. Eggermann, E., Bucurenciu, I., Goswami, S. P. $\delta$ Jonas, P. Nanodomain coupling between $\mathrm{Ca}^{2+}$ channels and sensors of exocytosis at fast mammalian synapses. Nature Rev. Neurosci. 13 7-21 (2012).

41. Felmy, F., Neher, E. \& Schneggenburger, R. Probing the intracellular calcium sensitivity of transmitter release during synaptic facilitation. Neuron 37 , 801-811 (2003).

42. Cooke, J. D., Okamoto, K. \& Quastel, D. M. The role of calcium in depolarization-secretion coupling at the motor nerve terminal. J. Physiol. 228, 459-497 (1973).

43. Llinas, R., Steinberg, I. Z. \& Walton, K. Relationship between presynaptic calcium current and postsynaptic potential in squid giant synapse. Biophys. J. 33, 323-351 (1981)

44. Hochner, B., Parnas, H. \& Parnas, I. Membrane depolarization evokes neurotransmitter release in the absence of calcium entry. Nature 342, 433-435 (1989).

45. Zhang, C. \& Zhou, Z. Ca ${ }^{2+-i n d e p e n d e n t ~ b u t ~ v o l t a g e-~}$ dependent secretion in mammalian dorsal root ganglion neurons. Nature Neurosci. 5, 425-430 (2002).

46. Mochida, S., Yokoyama, C. T., Kim, D. K., Itoh, K. \& Catterall, W. A. Evidence for a voltage-dependent enhancement of neurotransmitter release mediated via the synaptic protein interaction site of $\mathrm{N}$-type $\mathrm{Ca}^{2+}$ channels. Proc. Natl Acad. Sci. USA 95. 14523-14528 (1998).

47. Felmy, F., Neher, E. $\&$ Schneggenburger, R. The timing of phasic transmitter release is $\mathrm{Ca}^{2+}$-dependent and lacks a direct influence of presynaptic membrane potential. Proc. Natl Acad. Sci. USA 100. 15200-15205 (2003). 
48. Fili, O. et al. Direct interaction of a brain voltage-gated $\mathrm{K}^{+}$channel with syntaxin $1 \mathrm{~A}$ : functional impact on channel gating. J. Neurosci. 21, 1964-1974 (2001).

49. Feinshreiber, L., Singer-Lahat, D., Ashery, U. \&

Lotan, I. Voltage-gated potassium channel as a facilitator of exocytosis. Ann. NY Acad. Sci. 1152 87-92 (2009).

50. Feinshreiber, L. et al. Non-conducting function of the Kv2.1 channel enables it to recruit vesicles for release in neuroendocrine and nerve cells. J. Cell Sci. 123, 1940-1947 (2010).

51. Parnas, H. \& Parnas, I. The chemical synapse goes electric: $\mathrm{Ca}^{2+-}$ and voltage-sensitive GPCRs control neurotransmitter release. Trends Neurosci. 30, 54-61 (2007).

52. Kupchik, Y. M. et al. A novel fast mechanism for GPCR-mediated signal transduction - control of neurotransmitter release. J. Cell Biol. 192, 137-151 (2011).

53. Linial, M., llouz, N. \& Parnas, H. Voltage-dependent interaction between the muscarinic ACh receptor and proteins of the exocytic machinery. J. Physiol. 504, 251-258 (1997)

54. Ilouz, N., Branski, L., Parnis, J., Parnas, H. \& Linial, M. Depolarization affects the binding properties of muscarinic acetylcholine receptors and their interaction with proteins of the exocytic apparatus. J. Biol. Chem. 274, 29519-29528 (1999).

55. Kupchik, Y. M. et al. Molecular mechanisms that control initiation and termination of physiological depolarization-evoked transmitter release. Proc. Nat Acad. Sci. USA 105, 4435-4440 (2008).

56. Ohana, L., Barchad, O., Parnas, I. \& Parnas, H. The metabotropic glutamate G-protein-coupled receptors mGluR3 and mGluR $1 \mathrm{a}$ are voltage-sensitive. J. Biol. Chem. 281, 24204-24215 (2006)

57. Thompson, S. M. \& Gahwiler, B. H. Activity-dependent disinhibition. III. Desensitization \& GABAB receptormediated presynaptic inhibition in the hippocampus in vitro. J. Neurophysiol. 61, 524-533 (1989).

58. Kamiya, H., Shinozaki, H. \& Yamamoto, C. Activation of metabotropic glutamate receptor type $2 / 3$ suppresses transmission at rat hippocampal mossy fibre synapses. J. Physiol. 493, 447-455 (1996).

59. Moore, K. A., Nicoll, R. A. \& Schmitz, D. Adenosine gates synaptic plasticity at hippocampal mossy fiber synapses. Proc. Natl Acad. Sci. USA 100, 14397-14402 (2003)

60. He L \& Wu, L The debate on the kiss-and-run fusion at synapses. Trends Neurosci. 30, 447-455 (2007).

61. Richards, D. A. Vesicular release mode shapes the postsynaptic response at hippocampal synapses. J. Physiol. 587, 5073-5080 (2009).

62. Zhang, Q., Li, Y. \& Tsien, R. W. The dynamic control of kiss-and-run and vesicular reuse probed with single nanoparticles. Science 323, 1448-1453 (2009).

63. Blackmer, T. et al. G protein $\beta \gamma$ subunit-mediated presynaptic inhibition: regulation of exocytotic fusion downstream of $\mathrm{Ca}^{2+}$ entry. Science 292, 293-297 (2001).

64. Chen, X. K. et al. Activation of GPCRs modulates quantal size in chromaffin cells through $\mathrm{G}_{\beta v}$ and $\mathrm{PKC}$. Nature Neurosci. 8, 1160-1168 (2005).

65. Bucurenciu, I., Bischofberger, J. \& Jonas, P. A small number of open $\mathrm{Ca}^{2+}$ channels trigger transmitter release at a central GABAergic synapse. Nature Neurosci. 13, 19-21 (2010)

66. Fedchyshyn, M. J. \& Wang L. Y Developmental transformation of the release modality at the calyx of Held synapse. J. Neurosci. 25, 4131-4140 (2005).

Acknowledgements

The authors are supported by INSERM, Aix-Marseille Université, Centre National de la Recherche Scientifique, Agence Nationale de la Recherche (ANR 11 BSV4 016 01), Fondation pour le Recherche Medicale and the French Ministry of Research. We thank H. Alle, O. El Far, J.-M. Goaillard, V. Marra, M. Seagar and the reviewers for helpful discussion and comments on the manuscript.

\section{Competing interests statement}

The authors declare no competing financial interests.

\section{FURTHER INFORMATION}

